\title{
Driven colloidal fluids: construction of dynamical density functional theories from exactly solvable limits
}

\author{
Alberto Scacchi ${ }^{1}$, Matthias Krüger ${ }^{2}$ and Joseph $\mathrm{M}_{\text {Brader }}{ }^{1}$ \\ ${ }^{1}$ Department of Physics, University of Fribourg, CH-1700 Fribourg, Switzerland \\ 2 4th Institute for Theoretical Physics, Universität Stuttgart, and Max Planck Institute \\ for Intelligent Systems, Stuttgart, Germany
}

\begin{abstract}
The classical dynamical density functional theory (DDFT) provides an approximate extension of equilibrium DFT to treat nonequilibrium systems subject to Brownian dynamics. However, the method fails when applied to driven systems, such as sheared colloidal dispersions.

The breakdown of DDFT can be traced back to an inadequate treatment of the flowinduced distortion of the pair correlation functions. By considering the distortion of the pair correlations to second order in the flow-rate we show how to systematically correct the DDFT for driven systems. As an application of our approach we consider Poiseuille flow. The theory predicts that the particles will accumulate in spatial regions where the local shear rate is small, an effect known as shear-induced migration. We compare these predictions to Brownian dynamics simulations with generally good agreement.
\end{abstract}

Keywords: density functional theory, migration, nonequilibrium, shear

(Some figures may appear in colour only in the online journal)

\section{Introduction}

The response of particles in suspension to externally applied driving forces is a subject of considerable practical interest [1-3]. For example, many industrial processes involve the transport of micron or sub-micron sized particles along tubes or channels, as is the case in microfluidics, or require the particles to be dispersed by stirring or shearing. In addition, colloidal suspensions are very appealing for fundamental research, because they present relatively simple model system with length- and time-scales which enable basic aspects of nonequilibrium statistical mechanics to be studied in detail.

The classical dynamical density functional theory (DDFT) is a simple and popular method for predicting the time evolution of the one-body density of Brownian many-body systems in the presence of time-dependent external force fields [4]. In the simplest case this time-dependence can correspond to an instantaneous switching of the external potential from one form to another (e.g. switching off a confining harmonic potential), leading to a relaxational dynamics. However, in general, when including external forces, the dynamics might crucially depend on particle interactions, and e.g. change pair correlations in nontrivial ways, and it is in such situations that the inaccuracy of DDFT becomes apparent.

The failure of DDFT to describe certain aspects of driven systems arises from the absence of non-affine motion, which prevents the particles from following the externally prescribed flow field. The neglect of non-affine motion arises from the assumption that the two-body density relaxes instantaneously to that of the equilibrium system corresponding to the nonequilibrium density: the adiabatic approximation. This situation has been addressed in several previous studies $[5,6,8]$, all of which employ phenomenological corrections to DDFT in an effort to restore some aspects of the non-affine motion. In [5] and [6] an additional mean-field contribution to the particle current takes the form of a convolution between the density and a 'flow kernel', which captures how the particles roll past one another in the presence of a flow field. In [7] the general mathematical structure of the phenomenological theory was justified by showing how consideration of the flow-distorted pair correlations can generate non-affine current contributions when substituted into the formally exact equation of motion for the density. 
In this paper we develop a systematic scheme for describing external drive in DDFT by incorporating into the theory the influence on the one-body density of the distorted pair correlations to second order in the flow-rate. This progress builds upon the work of Brady and Vicic [9], who solved analytically the low-density pair Smoluchowski equation in a series expansion up to second order in the flow-rate. This result, which is valid for an arbitrary incompressible flow, contains the key physical feature missing from the standard formulation of DDFT, namely the influence of normal stresses on the one-body density. In all theoretical work so far performed only purely repulsive interparticle interactions have been considered. The results we present here are valid for arbitrary interactions, both attractive and repulsive. As an application of our theory we consider the shear-induced migration of repulsive colloidal particles subject to Poiseuille flow. As a consequence of the spatially varying shear rate the particles tend to drift towards regions where the spatial gradient of the shear rate is small.

The paper will be organized as follows: in section II.A we recall the standard DDFT, in section II.B we discuss the pair Smoluchowski equation for the two-body correlations and in section II.C give the analytic solution up to quadratic order in the flow-rate. In section II.D we specialize the results of the preceeding section to hard-spheres in three dimensions. In section II.E we exploit the solution of the pair Smoluchowski equation to systematically extend the DDFT equations to treat driven systems. In section III we apply the theory to treat Poiseuille flow and, finally, in section IV we provide comments and an outlook for future work.

\section{Theory}

\section{II.A. Dynamical density functional theory}

We consider a system of $N$ Brownian particles in an external potential field, $V_{\text {ext }}(\mathbf{r})$, interacting via the pair potential, $u(r)$. In addition to the external potential field the particles also experience a velocity field $\mathbf{v}(\mathbf{r})$ which represents the external driving (e.g. shear). The time evolution of the $N$-particle configurational probability distribution is given by the Smoluchowski equation [1]. Integration of the Smoluchowski equation over $N-1$ particle coordinates yields an exact equation of motion for the one-body density

$$
\begin{aligned}
& \frac{\partial \rho\left(\mathbf{r}_{1}, t\right)}{\partial t}+\nabla_{1} \cdot\left(\rho\left(\mathbf{r}_{1}, t\right) \mathbf{v}\left(\mathbf{r}_{1}, t\right)\right) \\
& =D_{0} \nabla_{1} \cdot \rho\left(\mathbf{r}_{1}, t\right)\left(\nabla_{1} \ln \rho\left(\mathbf{r}_{1}, t\right)+\nabla_{1} \beta V_{\mathrm{ext}}\left(\mathbf{r}_{1}, t\right)\right. \\
& \left.\quad+\int \mathrm{d} \mathbf{r}_{2} \rho_{0}^{(2)}\left(\mathbf{r}_{2}, t \mid \mathbf{r}_{1}\right) \nabla_{1} \beta u\left(r_{12}\right)\right),
\end{aligned}
$$

where $D_{0}$ is the bare diffusion coefficient and $\beta=\left(k_{\mathrm{B}} T\right)^{-1}$. The conditional two-body density, $\rho_{0}^{(2)}\left(\mathbf{r}_{2}, t \mid \mathbf{r}_{1}\right)$, is the density at $\mathbf{r}_{2}$, given that a particle is located at $\mathbf{r}_{1}$, and is related to the full twobody density according to $\rho_{0}^{(2)}\left(\mathbf{r}_{2}, t \mid \mathbf{r}_{1}\right)=\rho^{(2)}\left(\mathbf{r}_{1}, \mathbf{r}_{2}, t\right) / \rho\left(\mathbf{r}_{1}, t\right)$. Due to the presence of the conditional pair density equation (1) is not closed, but rather the first in a hierarchy.
In equilibrium the following sum-rule holds

$$
\int \mathrm{d} \mathbf{r}_{2} \frac{\rho_{\mathrm{eq}}^{(2)}\left(\mathbf{r}_{1}, \mathbf{r}_{2}\right)}{\rho\left(\mathbf{r}_{1}\right)} \nabla_{1} u\left(r_{12}\right)=\nabla_{1} \frac{\delta \mathcal{F}_{\mathrm{ex}}[\rho]}{\delta \rho\left(\mathbf{r}_{1}\right)},
$$

where $\mathcal{F}_{\text {ex }}[\rho]$ is the excess Helmholtz free energy functional $[11,12]$, which encodes the interaction potential. Making the (adiabatic) approximation that (2) holds also in nonequilibrium generates the standard DDFT

$$
\begin{aligned}
\frac{\partial \rho(\mathbf{r}, t)}{\partial t} & +\nabla \cdot(\rho(\mathbf{r}, t) \mathbf{v}(\mathbf{r}, t)) \\
& =D_{0} \nabla \cdot\left(\rho(\mathbf{r}, t) \nabla \frac{\delta \beta \mathcal{F}[\rho(\mathbf{r}, t)]}{\delta \rho(\mathbf{r}, t)}\right),
\end{aligned}
$$

where the total Helmholtz free energy $\mathcal{F}[\rho(\mathbf{r}, t)]$ is related to the excess part according to

$$
\mathcal{F}[\rho]=\mathcal{F}_{\text {id }}[\rho]+\mathcal{F}_{\text {ex }}[\rho]+\int \mathrm{d} \mathbf{r} V_{\text {ext }}(\mathbf{r}) \rho(\mathbf{r}, t),
$$

where the ideal contribution is given exactly by (the thermal wavelength $\Lambda$ is added for dimensional reasons)

$$
\mathcal{F}_{\text {id }}[\rho]=k_{\mathrm{B}} T \int \mathrm{d} \mathbf{r} \rho(\mathbf{r}, t)\left(\ln \left(\rho(\mathbf{r}, t) \Lambda^{3}\right)-1\right) .
$$

For situations where an external potential 'blocks' the affine flow (i.e. the particles cannot follow the flow into certain regions), then equation (3) can provide qualitatively correct results [13-16]. However, even in this case the errors can be quite large for interacting systems [16].

\section{II.B. Pair Smoluchowski equation}

We start from the exact solution of the underlying problem in the limit of small density and negligible external potential. Integration of the $N$-particle Smoluchowski equation over $N-2$ particles generates an exact equation for the nonequilibrium two-body density. In general this coarse-grained equation contains an integral term involving the three-body density [3]. However, in the low density limit this integral term can be neglected, leading to the following pair Smoluchowski equation

$$
\begin{aligned}
& \frac{\partial}{\partial t} \rho^{(2)}\left(\mathbf{r}_{1}, \mathbf{r}_{2}, t\right)+\sum_{i=1,2} \nabla_{i} \cdot\left(\rho^{(2)}\left(\mathbf{r}_{1}, \mathbf{r}_{2}, t\right) \mathbf{v}\left(\mathbf{r}_{i}, t\right)\right) \\
& =\sum_{i=1,2} \nabla_{i} \cdot\left(D _ { 0 } \rho ^ { ( 2 ) } ( \mathbf { r } _ { 1 } , \mathbf { r } _ { 2 } , t ) \nabla _ { i } \left(\ln \left(\rho^{(2)}\left(\mathbf{r}_{1}, \mathbf{r}_{2}, t\right)\right)\right.\right. \\
& \left.\left.\quad+\beta V_{\text {ext }}\left(\mathbf{r}_{i}, t\right)+\beta u\left(r_{12}\right)\right)\right),
\end{aligned}
$$

where the dependence of the pair density on two spatial arguments reflects the fact that an external field breaks translational invariance. When supplemented with appropriate boundary conditions, equations (1) and (6) form a closed set of equations for determining the dynamics of the one- and two-body density for low-density systems.

For a translationally invariant system the pair density becomes a function of the relative particle position, $\mathbf{r}=\mathbf{r}_{1}-\mathbf{r}_{2}$, and the pair correlations may be described by the radial distribution function, $g(\mathbf{r}, t) \equiv \rho^{(2)}(\mathbf{r}, t) / \rho_{b}^{2}$, where $\rho_{b}$ is the bulk 
density. Note that the radial distribution function can still be anisotropic due to the external flow field. In this translationally invariant situation the low density behaviour of $g(\mathbf{r}, t)$ is determined by the simpler pair Smoluchowski equation

$$
\begin{aligned}
& \frac{\partial g(\mathbf{r}, t)}{\partial t}+\nabla \cdot(g(\mathbf{r}, t) \mathbf{v}(\mathbf{r}, t)) \\
& \quad=\nabla \cdot\left(2 D_{0} \nabla g(\mathbf{r}, t)+2 D_{0}(\nabla \beta u(r)) g(\mathbf{r}, t)\right)
\end{aligned}
$$

where $\mathbf{r}=\mathbf{r}_{1}-\mathbf{r}_{2}$ and the $\nabla$ is taken with respect to $\mathbf{r}$. The velocity vector $\mathbf{v}$ refers here to the relative velocity of the two particles resulting from affine motion, $\mathbf{v}(\mathbf{r}, t)=\mathbf{v}\left(\mathbf{r}_{1}, t\right)-\mathbf{v}\left(\mathbf{r}_{2}, t\right)$. Equation (7) is valid for low $\rho_{b}$, but for all velocities and flow types (both compressible and incompressible). In the present work we will focus on low velocities (on the Brownian time scale) and incompressible flow, for which $\nabla \cdot \mathbf{v}(\mathbf{r})=0$.

\section{II.C. Exact solutions of the pair Smoluchowski equation for low flow-rates}

In sections II.C and II.E we will follow closely [9]. For a translationally invariant bulk system the steady-state distorted radial distribution function is given by the well-known result (see e.g. [9])

$$
g(\mathbf{r})=g_{\mathrm{eq}}(r)\left(1-\frac{R^{2}}{2 D_{0}}(\hat{\mathbf{r}} \cdot \mathbf{E} \cdot \hat{\mathbf{r}}) h_{1}(r)\right)
$$

to leading order in the flow rate. In this expression $\mathbf{E}=\left((\nabla \mathbf{v})+(\nabla \mathbf{v})^{T}\right) / 2$ is the symmetrized velocity gradient tensor, $g_{\mathrm{eq}}(r)=\exp (-\beta u(r))$ is the low density equilibrium radial distribution function, $\hat{\mathbf{r}}$ is a unit vector and $R$ is the particle radius. The dimensionless quantity $h_{1}(r)$ is a function which depends only on the radial coordinate, such that all anisotropy is captured by the quadratic form $\hat{\mathbf{r}} \cdot \mathbf{E} \cdot \hat{\mathbf{r}}$. The second term in (8) gives the leading order flow-induced distortion of the radial distribution function and is valid for all incompressible flows (for generalization to compressible flow see [18]). In appendix A we show that substitution of (8) into (7) leads to the requirement that $h_{1}(r)$ satisfies the following differential equation, where we use $\tilde{r}=r / R$ for brevity

$\frac{1}{\tilde{r}^{2}} \frac{\mathrm{d}}{\mathrm{d} \tilde{r}}\left(\tilde{r}^{2} g_{\mathrm{eq}}(r) \frac{\mathrm{d} h_{1}(r)}{\mathrm{d} \tilde{r}}\right)-\frac{6 g_{\mathrm{eq}}(r) h_{1}(r)}{\tilde{r}^{2}}=-\tilde{r} \frac{\mathrm{d} g_{\mathrm{eq}}(r)}{\mathrm{d} \tilde{r}}$,

where the pair potential enters equation (8) implicitly via $g_{\text {eq }}(r)$. The boundary conditions for this equation are: (i) that $h_{1}(r)$ vanishes as $r \rightarrow \infty$ and (ii) that the radial flux vanishes at short separations, to prevent the particles interpenetrating. For hard-core particles the second condition translates to $h^{\prime}(2 R)=-1$, where here and in the following $h^{\prime}$ denotes the derivative with respect to $\tilde{r}$.

We note that an alternative method of solving the pair Smoluchowski equation was considered in [10], where the authors employed multipole methods to expand the solution in spherical harmonics. Solutions were obtained numerically by truncating the harmonic series once a suitable convergence criterion had been satisfied.
For hard spheres there is a simple analytical solution of (9). At low density the equilibrium radial distribution function becomes a step function, $g_{\text {eq }}(r)=\Theta(2 R-|y|)$, where $\Theta(\cdot)$ is the step function (zero for negative arguments, unity for positive arguments). We thus obtain

$$
h_{1}^{\mathrm{hs}}(r)=\frac{16}{3 \tilde{r}^{3}} .
$$

Knowledge of $h_{1}^{\text {hs }}(r)$ for $r<2 R$ is not required, because the prefactor $g_{\text {eq }}(r)$ appearing for $r \geqslant 2 R$ in (8) is zero within this range. For other interaction potentials (9) must be solved numerically, although we are only aware of one study in which numerical solutions were investigated (for charged hard-spheres, see p 489 of [17]).

Equation (1) has been extended to second order in the flow-rate by Brady and Vicic [9]. Beyond second order the boundary layer which develops close to contact leads to fractional powers in the flow-rate expansion and calculations become considerably more complicated. Fortunately, all of the physics we are presently interested in occurs already at second order in flow-rate. The second order result is given by

$$
\begin{aligned}
g(\mathbf{r})= & g_{\mathrm{eq}}(r)\left(1-\frac{R^{2}}{D_{0}}(\hat{\mathbf{r}} \cdot \mathbf{E} \cdot \hat{\mathbf{r}}) h_{1}(r)\right. \\
& -\frac{R^{4}}{D_{0}^{2}}(\hat{\mathbf{r}} \cdot \mathbf{E} \cdot \hat{\mathbf{r}})^{2} h_{2}(r)-\frac{R^{4}}{D_{0}^{2}}(\hat{\mathbf{r}} \cdot \mathbf{E} \cdot \Omega \cdot \hat{\mathbf{r}}) h_{3}(r) \\
& \left.-\frac{R^{4}}{D_{0}^{2}}(\hat{\mathbf{r}} \cdot \mathbf{E} \cdot \mathbf{E} \cdot \hat{\mathbf{r}}) h_{4}(r)-\frac{R^{4}}{D_{0}^{2}}(\mathbf{E}: \mathbf{E}) h_{5}(r)\right)
\end{aligned}
$$

where the vorticity tensor is given by $\Omega=\left((\nabla \mathbf{v})-(\nabla \mathbf{v})^{T}\right) / 2$ and the double dot notation in the final term implies a full contraction over tensor indices. The second order contribution is constructed by considering all quadratic combinations of the fundamental flow tensors, $\mathbf{E}$ and $\Omega$, which describe the pure straining motion and the pure rotation, respectively. The fact that $\Omega: \Omega=\mathbf{E}: \mathbf{E}$ makes inclusion of an extra term proportional to $\Omega: \Omega$ unnecessary and $(\hat{\mathbf{r}} \cdot \Omega \cdot \hat{\mathbf{r}})^{2}$ is absent, because $\hat{\mathbf{r}} \cdot \Omega \cdot \hat{\mathbf{r}}=0$.

As previously, the function $h_{1}(r)$ is given by solution of (9). The four new, dimensionless radial functions, $h_{i}(r)$, $i=2, \ldots 5$, are given by solution of the equations (recall that primes denote derivatives with respect to $\tilde{r}$ )

$$
\begin{aligned}
& \frac{\mathrm{d}}{\mathrm{d} \tilde{r}}\left(\tilde{r}^{2} g_{0}(r) \frac{\mathrm{d}}{\mathrm{d} \tilde{r}} h_{2}(r)\right)-20 g_{0}(r) h_{2}(r) \\
& \quad=-\tilde{r}^{2} g_{0}(r) h_{1}(r)+\frac{1}{2} \tilde{r}^{3} g_{0}^{\prime}(r) h_{1}(r)+\frac{1}{2} \tilde{r}^{3} g_{0}(r) h_{1}^{\prime}(r), \\
& \frac{\mathrm{d}}{\mathrm{d} \tilde{r}}\left(\tilde{r}^{2} g_{0}(r) \frac{\mathrm{d}}{\mathrm{d} \tilde{r}} h_{3}(r)\right)-6 g_{0}(r) h_{3}(r)=-\tilde{r}^{2} h_{1}(r), \\
& \frac{\mathrm{d}}{\mathrm{d} \tilde{r}}\left(\tilde{r}^{2} g_{0}(r) \frac{\mathrm{d}}{\mathrm{d} \tilde{r}} h_{4}(r)\right)-6 g_{0}(r) h_{4}(r)=-8 h_{2}(r)+\tilde{r}^{2} h_{1}(r),
\end{aligned}
$$




$$
\frac{\mathrm{d}}{\mathrm{d} \tilde{r}}\left(\tilde{r}^{2} g_{0}(r) \frac{\mathrm{d}}{\mathrm{d} \tilde{r}} h_{5}(r)\right)=-2 h_{4}(r) .
$$

(Note that in [9] there is a typographical error on the rhs of equation (14). The version given here is the correct one.) Equations (9) and (12)-(15) can be found by substitution of (11) into (7) and equating terms with common tensorial prefactors, in the spirit of the calcuation presented in appendix A. Although equations (9) and (12)-(15) are coupled, they have the convenient property that determination of $h_{n}(r)$ only requires knowledge of other $h_{i}(r)$ for $i<n$. For the case of particles with a hard-core at small separations (the only kind we will consider in this work) the boundary conditions are that $h_{i}(r \rightarrow \infty) \rightarrow 0$ for all $i$ values and that

$$
\begin{gathered}
h_{1}^{\prime}(2 R)=-1 \\
h_{2}^{\prime}(2 R)=h_{1}(2 R) \\
h_{i}^{\prime}(2 R)=0 \quad i \geqslant 3,
\end{gathered}
$$

which arise from the boundary condition, $\mathbf{J}(\mathbf{r}) \cdot \hat{\mathbf{r}}=0$ at $\tilde{r}=2$, where $\mathbf{J}(\mathbf{r})$ is the current. For such boundary conditions the shooting method provides a simple and reliable numerical approach for obtaining solutions.

For the special case of hard spheres the solutions of equations (12)-(15) which satisfy the boundary conditions are

$$
\begin{gathered}
h_{2}^{\mathrm{hs}}(r)=\frac{2}{3}\left(\frac{1}{\tilde{r}}-\frac{16}{\tilde{r}^{5}}\right), \\
h_{3}^{\mathrm{hs}}(r)=\frac{8}{27}\left(\frac{3}{\tilde{r}}-\frac{4}{\tilde{r}^{3}}\right), \\
h_{4}^{\mathrm{hs}}(r)=-\frac{32}{63}\left(\frac{5}{\tilde{r}^{3}}-\frac{12}{\tilde{r}^{5}}\right), \\
h_{5}^{\mathrm{hs}}(r)=-\frac{4}{945}\left(\frac{105}{\tilde{r}}-\frac{200}{\tilde{r}^{3}}+\frac{144}{\tilde{r}^{5}}\right) .
\end{gathered}
$$

Each of these expressions is valid for $r \geqslant 2 R$ and, as was the case for $h_{1}(r)$, information for shorter separations is not required.

The distorted pair correlations provide information about the relative spatial distribution of the particles and thus enable (for pairwise additive potentials) the stress tensor to be calculated according to

$$
\boldsymbol{\sigma}=-k_{\mathrm{B}} T \rho_{b} \mathbf{1}+\frac{1}{2} \rho_{b}^{2} \int \mathrm{d} \mathbf{r} \frac{\mathbf{r r}}{r} \frac{\partial u(r)}{\partial r} g(\mathbf{r}),
$$

which is valid for a translationally invariant bulk system. For inhomogeneous systems the stress tensor becomes a function of position

$$
\begin{aligned}
\boldsymbol{\sigma}(\mathbf{r})= & -k_{\mathrm{B}} T \rho_{b} \mathbf{1} \\
& +\frac{1}{2} \int_{0}^{1} \mathrm{~d} \lambda \int \mathrm{d} \mathbf{r}^{\prime} \frac{\mathbf{r}^{\prime} \mathbf{r}^{\prime}}{r^{\prime}} \frac{\partial u\left(r^{\prime}\right)}{\partial r^{\prime}} \rho^{(2)}\left(\mathbf{r}+(1-\lambda) \mathbf{r}^{\prime}, \mathbf{r}-\lambda \mathbf{r}^{\prime}\right) .
\end{aligned}
$$

A derivation of (24) can be found in [19] (p 162).

\section{II.D. Special case: hard-spheres under shear flow}

To make expression (11) more concrete, let us specify to the case of a shear flow, with flow in the $x$-direction and gradient in the $y$-direction. In this cartesian coordinate system the symmetrized velocity gradient tensor has components $E_{i j}=\dot{\gamma}\left(\delta_{i x} \delta_{j y}+\delta_{j x} \delta_{i y}\right)$, where $\dot{\gamma}$ is the shear-rate. Equation (11) then becomes

$$
\begin{aligned}
\frac{g(\mathbf{r})}{g_{\mathrm{eq}}(r)}= & 1-\mathrm{Pe} \frac{x y}{r^{2}} h_{1}(r)-\mathrm{Pe}^{2}\left(\frac{x^{2} y^{2}}{r^{4}} h_{2}(r)\right. \\
& \left.+\frac{\left(x^{2}-y^{2}\right)}{4 r^{2}} h_{3}(r)+\frac{\left(x^{2}+y^{2}\right)}{4 r^{2}} h_{4}(r)+\frac{1}{2} h_{5}(r)\right),
\end{aligned}
$$

where Pe $=\dot{\gamma} R^{2} / D_{0}$ is the Péclet number encoding the competition between shear motion and Brownian diffusion. For hard-spheres the $h_{i}$ are given by equations (10) and (19)-(22).

Substituting equation (25) into (23) yields the pair interaction contribution (omitting zero and one-body terms) to the shear viscosity

$$
\eta=\eta_{s} \frac{12}{5} \phi^{2}
$$

where $\eta=\sigma_{\mathrm{xy}} / \dot{\gamma}, \eta_{s}=k_{\mathrm{B}} T /\left(6 \pi D_{0} R\right)$ is the solvent viscosity and $\phi=4 \pi R^{3} \rho_{b} / 3$ is the volume fraction [22]. Due to symmetry, contributions to $g(\mathbf{r})$ of second order in the flow-rate do not contribute to the shear viscosity. However, they do contribute to the normal stresses and suspension pressure. The interaction contributions to the first normal stress difference, $N_{1}=\sigma_{\mathrm{xx}}-\sigma_{\mathrm{yy}}$, second normal stress difference, $N_{2}=\sigma_{\mathrm{yy}}-\sigma_{\mathrm{zz}}$, and pressure, $\Pi=-\operatorname{Tr} \sigma / 3$, are given by

$$
\begin{gathered}
N_{1}=\frac{k_{\mathrm{B}} T}{R^{3}} \frac{8}{45 \pi} \mathrm{Pe}^{2} \phi^{2}, \\
N_{2}=-\frac{k_{\mathrm{B}} T}{R^{3}} \frac{8}{63 \pi} \mathrm{Pe}^{2} \phi^{2}, \\
\Pi=\frac{k_{\mathrm{B}} T}{R^{3}} \frac{4}{15 \pi} \mathrm{Pe}^{2} \phi^{2} .
\end{gathered}
$$

In this work we seek to address a major failing of DDFT for driven systems by incorporating a mechanism for generating flux orthogonal to the direction of flow, arising from interparticle interactions, which will lead to nontrivial changes in the density. Such 'lateral fluxes' are closely related to the normal stress differences under flow and so it would seem that a requirement of a sensible DDFT extension is that the second order in flow-rate distortion of $g(\mathbf{r})$ be correctly handled.

\section{II.E. Application to DDFT}

II.E.1. General approximations leading to a DDFT. We now wish to exploit the exact limiting results from section II.C to approximate the integral term in (1) and thus arrive at a closed theory for the dynamics of the one-body density under external driving. The key result, equation (11), is strictly only valid for translationally invariant systems, while equation (1) 
requires as input the inhomogeneous nonequilibrium twobody density. In steady-state the simplest approximation for the inhomogeneous two-body density is

$$
\begin{aligned}
\rho_{0}^{(2)}\left(\mathbf{r}_{2} \mid \mathbf{r}_{1}\right)= & \rho_{0, \mathrm{eq}}^{(2)}\left(\mathbf{r}_{2} \mid \mathbf{r}_{1}\right)\left(1-\frac{R^{2}}{D_{0}}(\hat{\mathbf{r}} \cdot \mathbf{E} \cdot \hat{\mathbf{r}}) h_{1}(r)\right. \\
& -\frac{R^{4}}{D_{0}^{2}}(\hat{\mathbf{r}} \cdot \mathbf{E} \cdot \hat{\mathbf{r}})^{2} h_{2}(r)-\frac{R^{4}}{D_{0}^{2}}(\hat{\mathbf{r}} \cdot \mathbf{E} \cdot \Omega \cdot \hat{\mathbf{r}}) h_{3}(r) \\
& \left.-\frac{R^{4}}{D_{0}^{2}}(\hat{\mathbf{r}} \cdot \mathbf{E} \cdot \mathbf{E} \cdot \hat{\mathbf{r}}) h_{4}(r)-\frac{R^{4}}{D_{0}^{2}}(\mathbf{E}: \mathbf{E}) h_{5}(r)\right),
\end{aligned}
$$

where $\mathbf{r}=\mathbf{r}_{2}-\mathbf{r}_{1}$. This approximation is equivalent to writing $\rho_{0}^{(2)}\left(\mathbf{r}_{2} \mid \mathbf{r}_{1}\right)=\rho\left(\mathbf{r}_{2}\right) g(\mathbf{r})$, with $g(\mathbf{r})$ given by (11).

The approximate form (30) does not solve (6) exactly: substitution of (30) into (6) generates additional terms involving the product of flow rate and external field force of order $\dot{\gamma} \nabla V_{\text {ext }}(\mathbf{r})$. However, provided that these additional terms remain sufficiently small, the approximation (30) is justified. For example, in a low density system of hard spheres at a hard repulsive wall these contributions will only contribute when particles are in direct contact with the substrate. It would be desirable to have an exact expression for the nonequilibrium two-body density in inhomogeneous situations, but this is unfortunately not available at present. Jin et al have pointed out that incorporating density gradient terms in the expression for the distorted inhomogeneous pair correlation function is essential in recovering the proper behaviour of the mass diffusion coefficient. Due to the simplicity of our approximation (30) our theory may fail on this point.

We note, importantly, that the approximation in equation (30), applied for the case of a single wall, yields the correct value for the force exerted on the wall [7], see appendix C for details. For the special case of hard spheres at a hard wall, this implies that the contact density at the wall is found correctly, i.e. including its change due to shear.

If we accept (30) as a reasonable approximation, then it is still neccessary to determine an optimal choice for the equilibrium two-body density. In the context of DDFT the natural choice for the first term in (30) is the adiabatic pair density corresponding to the instantaneous one-body density. This choice has the convenient feature that the first term in (30) when substituted into (1) generates the DDFT.

Substitution of (30) into (1) and using (2) leads directly to the following generalized DDFT equation

$$
\begin{aligned}
\frac{\partial \rho\left(\mathbf{r}_{1}, t\right)}{\partial t} & +\nabla_{1} \cdot\left(\rho\left(\mathbf{r}_{1}, t\right)\left(\mathbf{v}\left(\mathbf{r}_{1}, t\right)+\mathbf{v}_{\mathrm{fl}}\left(\mathbf{r}_{1}, t\right)\right)\right) \\
& =D_{0} \nabla \cdot\left(\rho\left(\mathbf{r}_{1}, t\right) \nabla_{1} \frac{\delta \beta \mathcal{F}[\rho]}{\delta \rho\left(\mathbf{r}_{1}, t\right)}\right) .
\end{aligned}
$$

This expression has precisely the form given in references $[5,6]$, where it was proposed using physical arguments. The approximations used here are generally also in line with those proposed in [7]. The non-affine velocity contribution, $\mathbf{v}_{\mathrm{fl}}\left(\mathbf{r}_{1}, t\right)$, arising from the coupling of interparticle interactions with external flow, is given by

$$
\begin{aligned}
\mathbf{v}_{\mathrm{fl}}\left(\mathbf{r}_{1}, t\right)= & R^{2} \int \mathrm{d} \mathbf{r}_{2} \frac{\rho_{\mathrm{eq}}^{(2)}\left(\mathbf{r}_{1}, \mathbf{r}_{2}\right)}{\rho\left(\mathbf{r}_{1}\right)}\left(\left(\hat{\mathbf{r}}_{12} \cdot \mathbf{E} \cdot \hat{\mathbf{r}}_{12}\right) h_{1}\left(r_{12}\right)\right. \\
& +\frac{R^{2}}{D_{0}}\left[\left(\hat{\mathbf{r}}_{12} \cdot \mathbf{E} \cdot \hat{\mathbf{r}}_{12}\right)^{2} h_{2}\left(r_{12}\right)+\left(\hat{\mathbf{r}}_{12} \cdot \mathbf{E} \cdot \Omega \cdot \hat{\mathbf{r}}_{12}\right) h_{3}\left(r_{12}\right)\right. \\
& \left.\left.+\left(\hat{\mathbf{r}}_{12} \cdot \mathbf{E} \cdot \mathbf{E} \cdot \hat{\mathbf{r}}_{12}\right) h_{4}\left(r_{12}\right)+(\mathbf{E}: \mathbf{E}) h_{5}\left(r_{12}\right)\right]\right) \nabla_{1} \beta u\left(r_{12}\right) .
\end{aligned}
$$

This result is valid for any incompressible flow and arbitrary pair potential. The latter enters both explicitly via the force term $\nabla_{1} \beta u\left(r_{12}\right)$ and implicitly via the $h_{i}(r)$, which are given by solution of equations (9), (12)-(15). Despite our approximations equation (32) is still not tractable for practical applications, because knowledge of the pair density of the adiabatic state is required.

In order to obtain a theory which is closed on the level of the one-body density we factorize the two-body density

$$
\rho_{\mathrm{eq}}^{(2)}\left(\mathbf{r}_{1}, \mathbf{r}_{2}\right)=\rho\left(\mathbf{r}_{1}\right) \rho\left(\mathbf{r}_{2}\right) \exp \left(-\beta u\left(r_{12}\right)\right),
$$

consistent with the low density limit. Substituting (33) into (32) then generates a convolution form

$$
\mathbf{v}_{\mathrm{fl}}\left(\mathbf{r}_{1}, t\right)=\int \mathrm{d} \mathbf{r}_{2} \rho\left(\mathbf{r}_{2}\right) \mathbf{K}\left(r_{12}\right)
$$

where the (vectorial) flow kernel is given by

$$
\begin{aligned}
\mathbf{K}(\mathbf{r})= & -R^{2}\left[(\hat{\mathbf{r}} \cdot \mathbf{E} \cdot \hat{\mathbf{r}}) h_{1}(r)+\frac{R^{2}}{D_{0}}\left((\hat{\mathbf{r}} \cdot \mathbf{E} \cdot \hat{\mathbf{r}})^{2} h_{2}(r)\right.\right. \\
& +(\hat{\mathbf{r}} \cdot \mathbf{E} \cdot \Omega \cdot \hat{\mathbf{r}}) h_{3}(r)+(\hat{\mathbf{r}} \cdot \mathbf{E} \cdot \mathbf{E} \cdot \hat{\mathbf{r}}) h_{4}(r) \\
& \left.\left.+(\mathbf{E}: \mathbf{E}) h_{5}(r)\right)\right] \nabla \exp (-\beta u(r))
\end{aligned}
$$

Equations (31), (34) and (35) provide a closed theory for the dynamics of the one-body density, provided that one has access to a suitable approximation for the equilibrium free energy. The kernel (35) has been derived by considering the pair Smolochowski equation in the low desnity limit. When applying the theory at finite densities it may be useful to rescale the kernel using the contact value of the equilibrium radial distribution function, $g_{\mathrm{eq}}(2 R)$.

For the special case of shear flow, with flow in the $x$-direction and gradient in the $y$-direction, the kernel can be written

$$
\begin{aligned}
\mathbf{K}(\mathbf{r})= & -D_{0}\left(\operatorname{Pe} \frac{x y}{r^{2}} h_{1}(r)-\operatorname{Pe}^{2}\left(\frac{x^{2} y^{2}}{r^{4}} h_{2}(r)+\frac{\left(x^{2}-y^{2}\right)}{4 r^{2}} h_{3}(r)\right.\right. \\
& \left.\left.+\frac{\left(x^{2}+y^{2}\right)}{4 r^{2}} h_{4}(r)+\frac{1}{2} h_{5}(r)\right)\right) \nabla \exp (-\beta u(r)) .
\end{aligned}
$$

If the external field is such that the density only varies in the shear gradient direction, as is the case for channel-flow or shear parallel to a wall, the geometry of the situation allows the convolution integral (34) to be partially evaluated. The three dimensional convolution thus reduces to a one-dimensional convolution

$$
\mathbf{v}_{\mathrm{fl}}\left(y_{1}, t\right)=\int_{-\infty}^{\infty} \mathrm{d} y_{2} \rho\left(y_{2}\right) K_{y}\left(y_{1}-y_{2}\right),
$$


where $\mathrm{K}(y)$ is the resolved kernel required to treat the planar geometry. The non-affine velocity acts in the $y$-direction. In appendix B we show how the resolved kernel can be explicitly calculated for hard spheres under shear. The result is

$$
\begin{aligned}
\mathrm{K}_{y}^{\mathrm{hs}}(y)= & -\mathrm{Pe}^{2} \pi \frac{D_{0}}{R}\left(\frac{y}{2 R}\right)\left[-\frac{2}{27}\left(1-\frac{3 y^{2}}{(2 R)^{2}}\right)\right. \\
& \left.+\frac{2}{63}\left(1+\frac{y^{2}}{(2 R)^{2}}\right)+\frac{128}{945}\right] \Theta(2 R-|y|) .
\end{aligned}
$$

The functional form of the kernel is shown in the inset of figure 1 . The fact that the resolved kernel is antisymmetric in $y$ has the consequence that the density in bulk is not influenced by the flow, i.e. evaluation of the integral (37) for $\rho(y)=\rho_{b}$ yields zero. Moreover, for inhomogeneous systems the leading order flow-induced changes in the density are of order $\mathrm{Pe}^{2}$. This is consistent with the fact that the normal stresses driving lateral density currents are also second order in Pe.

For potentials consisting of a hard sphere repulsion and a longer range tail, e.g. the hard-core Yukawa potential, the Kernel splits into a sum of contact and tail contributions

$$
\mathrm{K}_{y}^{\mathrm{tot}}(y)=\mathrm{K}_{y}^{\mathrm{c}}(y)+\mathrm{K}_{y}^{\mathrm{t}}(y),
$$

where we only consider the $y$-component of the kernel. The contact contribution is given by

$$
\begin{aligned}
\mathrm{K}_{y}^{\mathrm{c}}(y)= & \frac{\mathrm{Pe}^{2} \pi D_{0}}{R}\left(\frac{y}{2 R}\right)\left[h_{2}(2 R) \frac{y^{2}}{(2 R)^{2}}\left(1-\frac{y^{2}}{(2 R)^{2}}\right)\right. \\
& -h_{3}(2 R) \frac{1}{4}\left(-1+\frac{3 y^{2}}{(2 R)^{2}}\right)+h_{4}(2 R) \frac{1}{4}\left(1+\frac{y^{2}}{(2 R)^{2}}\right) \\
& \left.+h_{5}(2 R)\right] \exp (-\beta u(2 R)) \Theta(2 R-|y|)
\end{aligned}
$$

where the functions $h_{2}(2 R) \ldots h_{5}(2 R)$ are now obtained from numerical solution of equations (12)-(15). The tail contribution in (39) is calculated by first expressing (36) in spherical polar coordinates (choosing the azimuthal angle $\phi$ around the $y$-axis, as described in appendix B) and then integrating over slices at fixed $y$. We thus obtain

$$
\begin{aligned}
\mathrm{K}_{y}^{\mathrm{c}}(y)= & \int_{\alpha(y)}^{\infty} \mathrm{d} r \frac{\mathrm{Pe}^{2} \pi D_{0}}{R^{2}} y\left[h_{2}(r) \frac{y^{2}}{r^{2}}\left(1-\frac{y^{2}}{r^{2}}\right)\right. \\
& -h_{3}(r) \frac{1}{4}\left(-1+\frac{3 y^{2}}{r^{2}}\right)+h_{4}(r) \frac{1}{4}\left(1+\frac{y^{2}}{r^{2}}\right) \\
& \left.+h_{5}(r)\right] \exp (-\beta u(r)) \Theta(2 R-|y|) .
\end{aligned}
$$

where the lower boundary is given by

$$
\alpha(y)= \begin{cases}2 R & |y| \leqslant 2 R \\ |y| & \text { otherwise }\end{cases}
$$

Due to the presence of the numerically determined $h$-functions, this integral must be numerically evaluated. Fortunately, this need only be performed once for any given interaction potential.

The expression we derive here for the flow kernel is based on the low density limiting solution of the pair Smoluchowski equation. When seeking to apply the theory to higher density systems the accuracy of the approach would probably be improved by scaling the kernel with the equilibrium contact value of the radial distribution function, $g_{\text {eq }}(2 R)$ for the pair potential of interest. We have have not done this here, because we only perform numerical calculations at low density statepoints for hard spheres (in which case $g_{\mathrm{eq}}(2 R) \sim 1$ ).

II.5.2. Numerical example for simple shear. As an initial test of our theory we consider the response of the density at a single soft wall to an imposed linear shear flow parallel to the wall. Because our theory is based on the pair Smoluchowski equation, which neglects three-body correlations, we deliberately choose a low density state point to be consistent with the underlying assumptions. Although we expect our approach to be useful also at higher densities, the approximations involved then become uncontrolled and difficult to assess. In figure 1 we show the steady-state density profiles of a low density suspension for four different values of Pe. In accordance with our previous findings based on a more empirical flow kernel approach [5], we find that the new kernel also predicts that shear flow parallel to the wall increases the density of particles at the wall. This occurs as particles are forced into the wall by collisions with their neighbours. This 'pile up' of density at the wall is associated with a pressure increase, relative to the equilibrium value (see appendix C).

\section{Application: shear-induced migration}

Microfluidic devices in which small quantities of fluid are driven through a microchannel are an emerging technology for processing suspensions (e.g. pharmaceuticals). If the shear-rate varies appreciably on the scale of a particle diameter then the particles undergo a biased diffusion which causes them to drift to regions of low shear gradient; a phenomenon known as shear-induced migration. The migration of colloids in channel flow is a dominant transport mechanism in suspensions which has been exploited to facilitate segregation in colloidal mixtures [26, 27] and which is relevant for understanding the flow of blood [27] as well as for numerous applications, such as food processing [28]. A variety of theoretical studies [29-32] and experiments [33-35] have addressed the migration dynamics of monodisperse hard-sphere colloids. However, the existing theoretical approaches remain on a semi-phenomenological level, where the interparticle interaction potential does not enter explicitly.

Recently, Jin et al have developed a theoretical approach to treat shear-gradient concentration coupling in colloidal dispersions under flow [36]. This study was motivated by the desire to understand the non-uniform flow and instabilities observed in colloidal glasses, although the approach could also be well applied to shear induced migration in liquid states, as considered in the present work. In common with our approach Jin et al start from the course grained Smoluchowski equation for the one-body density, however, the inhomogeneous pair correlations are treated quite differently. Whereas we consider distortions of adiabatic state pair correlations, which enables us to 


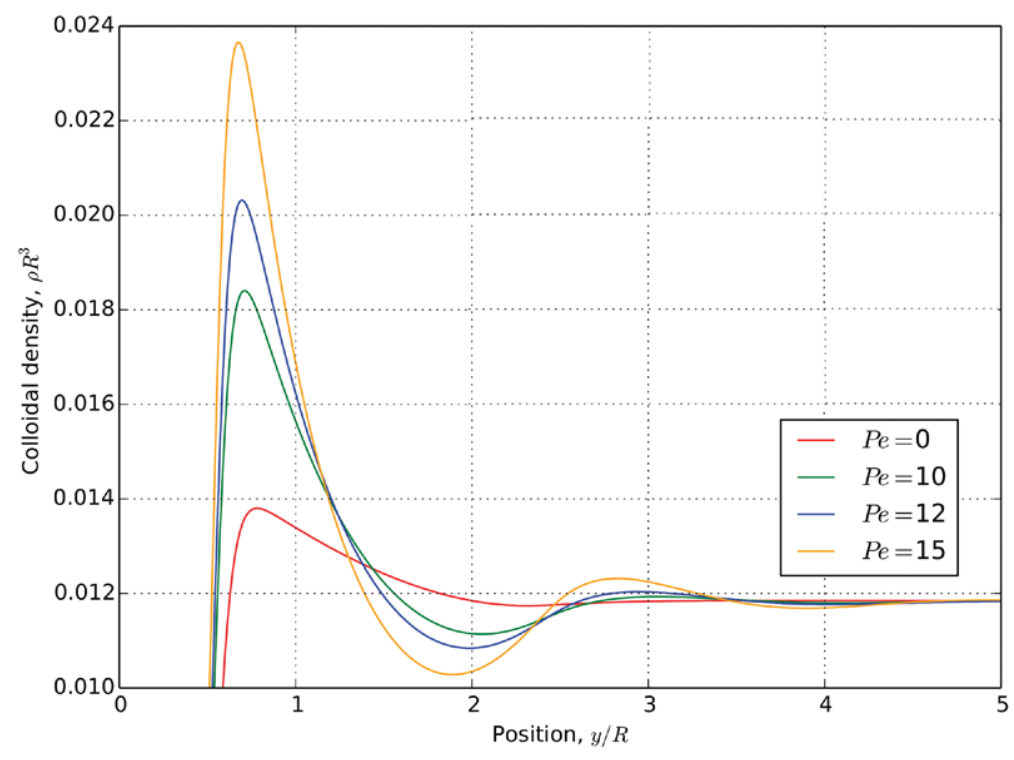

Figure 1. Density profiles of hard spheres at a wall from DDFT. The shear flow is applied parallel to the softly repulsive wall. The reduced bulk density is low, $\rho R^{3}=\simeq 0.01194$. Results are shown for four different values of Pe, as given in the legend. As Pe is increased the packing peak at the wall grows in amplitude as particles are forced into the wall by collisions with their neighbours.

employ a free energy functional, Jin et al approximate the full nonequilibrium pair correlation function directly. The latter method enables a more controlled treatment of the influence of density gradients on the distorted parts of the pair correlations, but prohibits connection to an equilibrium free energy.

To treat Poiseuille flow within our approach it is neccessary to first extend the theory of the flow kernel to treat spatial variations in the flow rate, i.e. spatial variations in $\mathbf{E}$ and $\Omega$. At the current level of development of our approach we are forced to make an ad hoc assumption about how this generalization may best be achieved. The Poiseuille flow to be implemented here is represented by the velocity field

$$
\mathbf{v}(y)=\mathbf{e}_{y} \frac{v_{0}}{2}\left(\frac{H^{2}}{4}-y^{2}\right) \Theta\left(\frac{H}{2}-|y|\right),
$$

where $H$ is the width of the channel to which the particles are confined and $v_{0}$ is the shear rate gradient of the flow profile. The maximal velocity occurs at the center of the channel and is given by $v_{\max }=v_{0} H^{2} / 8$, whereas the maximal shear rate is at the sides of the channel and is given by $\left|\dot{\gamma}_{\max }\right|=v_{0} H / 2$. Because the shear flow is parallel to the confining walls all contributions to the flow kernel linear in Pe vanish by symmetry. As we do not have exact results, even at low density, for the distortion of the two-body density induced by inhomogeneous flow (viz. spatially dependent shear rate) there is no unique choice how to generalize our second order results for the flow kernel. However, our numerical investigations have led us to the pragmatic solution of replacing the constant $\mathrm{Pe}^{2}$ appearing in equations (38)-(41) by a spatially dependent function, according to $\mathrm{Pe}^{2} \rightarrow \dot{\gamma}(y) \frac{\dot{\gamma}_{0} R^{4}}{D_{0}^{2}}$, where the spatially dependent shear rate is given by

$$
\dot{\gamma}(y)=-v_{0}|y| \text {, }
$$

We defer a full analysis of spatially dependent shear rates to a future work.

In order to solve the generalized DDFT equations (31), (37) and (38) for hard spheres we employ a softly repulsive external potential

$$
V_{\text {ext }}(y)=V_{0} \sum_{i=1,2} \exp \left(\frac{-\left(y-y_{i}\right)^{2}}{2 \alpha^{2}}\right),
$$

where $y_{1}$ and $y_{2}$ specify the positions of the walls, $V_{0}$ and $\alpha$ give the height and width of the wall potential. For our numerical DDFT calculations we choose $V_{0}=50 k_{\mathrm{B}} T$ and $\alpha=0.2 R$. In order to specify to hard spheres the Helmholtz free energy functional entering (31) must be specified. We choose to employ the standard Rosenfeld functional [37]. For the situations to be considered in this work the Rosenfeld functional provides very accurate results for the equilibrium density and should thus provide a good basis for calculations under shear.

Our density functional predictions are complemented by Brownian dynamics simulations of a system of 200 particles in a simulation box of side lengths $L_{y}=17.38 d$, $L_{x}=L_{z}=\sqrt{N / \rho L_{y}}=10.976 d$, where $d$ is the particle diameter. The algorithm employed is standard BD [38]. For practical reasons we consider pseudo hard spheres interacting via a WCA repulsive potential (cut and shifted Lennard-Jones, with the cut at $r=d$ ) with interaction strength $\varepsilon=0.01$. Periodic boundaries are used in the $x$ and $z$ directions. The particles move freely for values of $y$ between $y=-L_{y} / 2$ and $y=L_{y}$ 12 . However, if the particles leave this region they experience a soft confining repulsion: $F(y)=\epsilon_{w} \chi^{24} /\left(y+L_{y} / 2-\chi\right)^{24}$ for $y<-L_{y} / 2$ and $F(y)=-\epsilon_{w} \chi^{24} /\left(-y+L_{y} / 2+\chi\right)^{24}$ for $y>L_{y} / 2$. The parameter $\chi$ is set equal to $R$.

In figure 2 we address Poiseuille flow and show steadystate density profiles for four different values of the flow rate. 


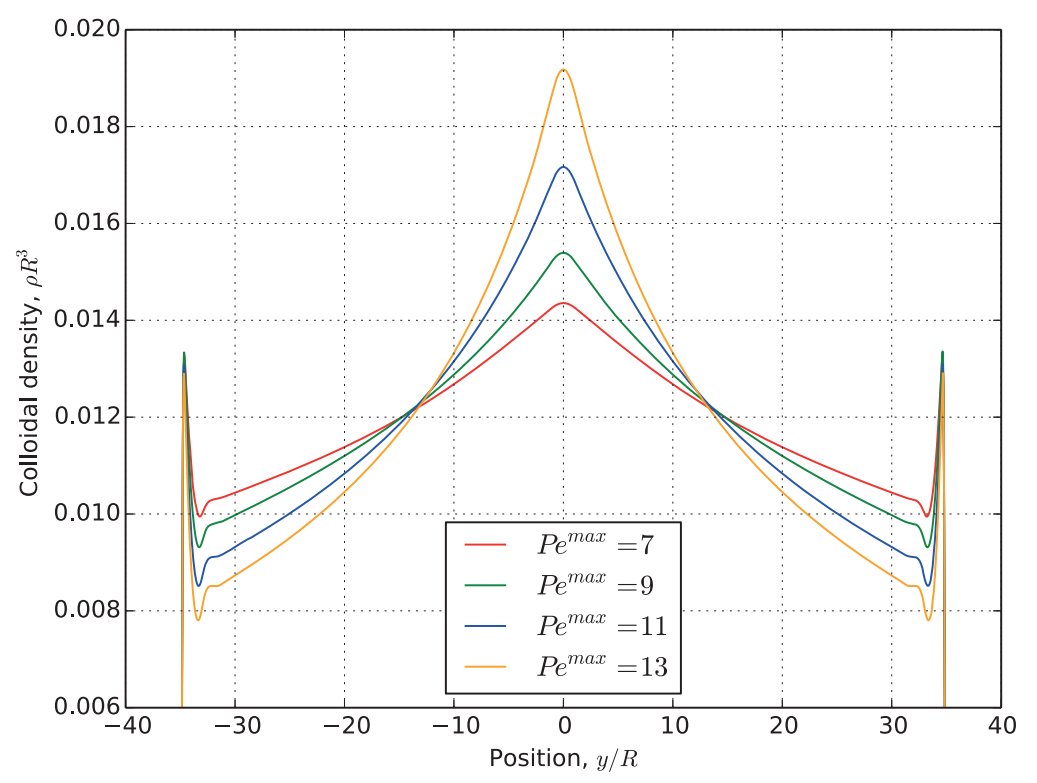

Figure 2. Steady-state density profiles calculated using DDFT for four different values of the maximal Péclet number (the Péclet number corresponding to the shear rate at the boundaries). As the flow rate is increased the central peak grows in magnitude as particles drift towards the centre, driven by collisions with their neighbours. This collision induced flux is balanced in steady-state by regular Brownian diffusion.

As the rate of flow is increased the particles begin to accumulate in the center of the channel. The physical reason for this is that particles which are not located at $y=0$ (the central plane through the channel) experience on average more collisions on one side than the other, due to the spatially varying shear rate in Poiseuille flow. The net result of these collisions is a biased diffusion, which tends to make the particles drift towards the center of the channel. Following the switching-on of the Poiseuille flow the density peak at the center develops until regular Brownian diffusion, which seeks to smooth density gradients, balances the biased diffusion induced by the inhomogeneous flow. A steady state density profile thus results. Although a systematic study of the transient development of the migration peak still needs to be performed we find that the time required for a steady state to develop is a strong function of the flow rate. We were thus careful that our calculations were of sufficient duration that a true steady-state solution is obtained.

In figure 3 we show Brownian dynamics results for the steady state density profiles of pseudo hard-spheres subject to Poiseuille flow. When performing our Brownian dynamics simulations we were careful only to collect data beyond the transient regime. The simulated density profiles exhibit the same characteristic triangular shape observed in our DDFT calculations. The particles which are transported by the migration mechanism to the center of the channel are consequently depleted from the regions close to the wall, leading to a reduction in packing effects in this region. In general we find that for the relatively narrow channels investigated in this work that there exists a competition between layering at the wall and the drift of particles to the center of the channel. This becomes more apparent when looking at the transient dynamics (not shown) - for short times after the switch on of flow the layering at the wall becomes more pronounced, but then decreases at later times as particles are transported towards the channel center, thus reducing the local density in the vicinity of the walls.

In figure 4 we show a fit of the simulation data obtained by adjusting the value of Pe employed in the DDFT, treating this as a free fit parameter. The fit was performed to obtain the best global representation of the simulation data, rather than aiming to match any specific feature (e.g. the height of the central peak). Although this initial result does not constitute a detailed comparison with Brownian dynamics simulations, it nevertheless demonstrates that our theory can be used to capture the qualitative form of the steady-state density profile. It is apparent that there is a significant mismatch between the Péclet number employed in the simulations and the value of Pe employed to fit our DDFT curve. This can be attributed to the following. (i) When generalizing our theory to treat Poiseuille flow, which has a spatially dependent shear rate, we were forced to make an uncontrolled approximation to extend a theory based on information obtained from homogeneous flow calculations. We believe that this step preserves the correct qualitative behaviour, but is quanitatively in error. Improving upon this point will be a subject of future research. (ii) In order to obtain a large migration peak in simulation, it was found neccessary to consider rather large values of Pe. We have then sought to reproduce these density profiles using a theory based on a series expansion of the density in powers of Pe. Quantitative agreement between simulation and experiment can thus only be expected at smaller values of of Pe. One could argue that applying our theory at large Pe violates the underlying assumptions of the approach, however, the fact that the simulation data can be rather well fit by using Pe as a free parameter suggests that the extended DDFT approach may well be of use in describing the density for a broad range of Pe. A more systematic comparison of our theory with Brownian dynamics simulation will be performed in the near future. 


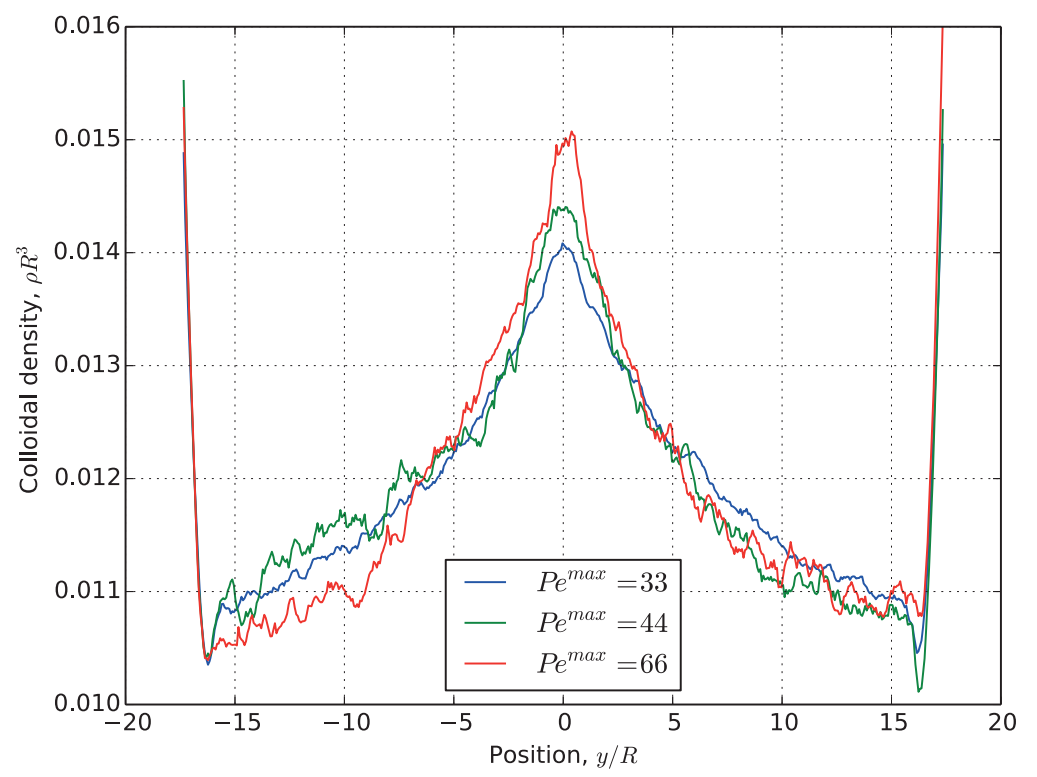

Figure 3. Brownian dynamics simulation results of shear migration for a system of pseudo-hard spheres whose peak in the density profile increases in magnitude.

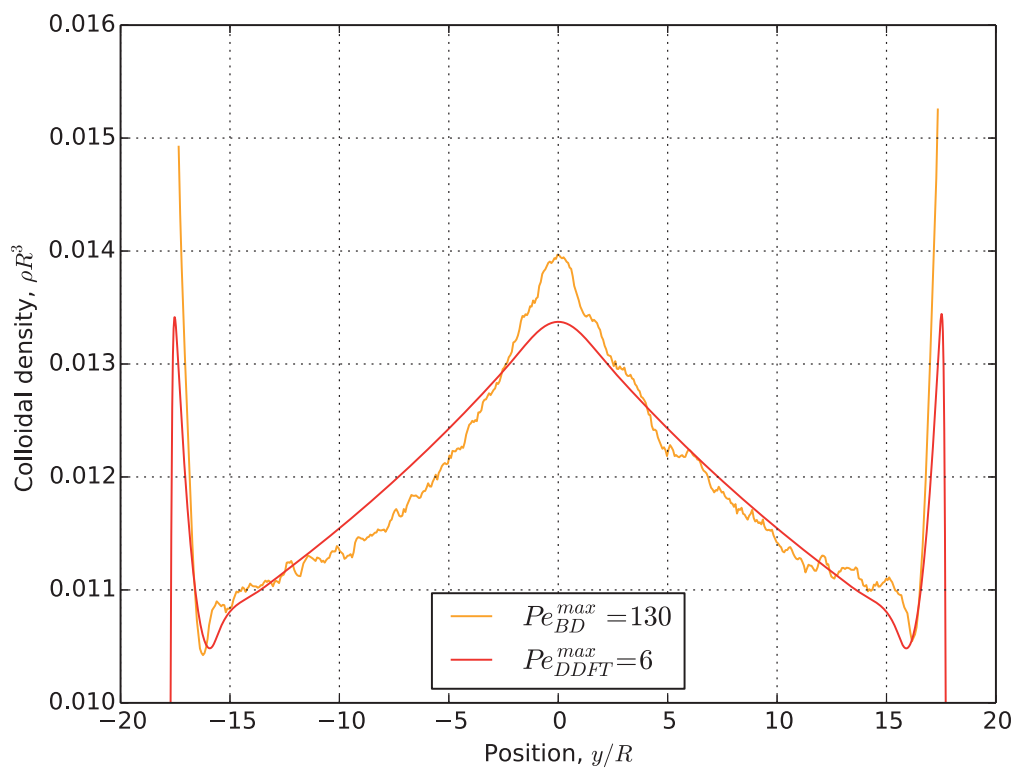

Figure 4. Fitting Brownian dynamics simulation data for the density profile of pseudo-hard spheres under Poiseuille flow using the modified DDFT theory developed in this work. The fit was performed to give the best global match between simulation and theory, where the $\mathrm{Pe}^{\mathrm{max}}$ number employed in the theory is treated as a fit parameter.

\section{Discussion}

In this paper we have presented a method by which DDFT may be corrected to incorporate the effects of external flow. The theory is in principle valid for all types of incompressible flow (e.g. shear or extensional) and contains corrections due to nonaffine particle motion up to second order in the flow rate. The most general form of the theory is given by (31) with nonaffine flow velocity given by (32). For the special case of hard spheres with shear flow in $x$-direction and gradient in $y$-direction the nonaffine velocity term simplifies to (38), where only the $y$-component of the kernel is of physical interest. The starting point of our theory is the exact equation of motion for the one-body density (1). Using exact results for the two-body density (at low density, to second order in Pe) we approximated the integral term in (1) to obtain a closed theory for the one-body density. We have thus demonstrated that the flowinduced distortion of the two-body density, usually neglected in DDFT treatments of driven systems, leads to an additional non-affine contribution to the particle current. When suitably approximated this non-affine contribution can be expressed as a convolution of the one-body density with a flow kernel.

We note that our nonequilibrium theory fits very well within the general framework of the fundamental measures theory (FMT) approach to the DFT of hard particle fluids [37]. Within our dynamic theory the usual set of geometric weight functions employed in the FMT is supplemented by a nonequilibrium weight function, the flow kernel, which generates 
lateral fluxes arising from non-affine motion. The difficulty of implementation of our theory is thus on the same level as standard DDFT for hard spheres, requiring only one additional convolution at each time-step.

As an application of our theory to a physically relevant problem we have considered the shear-induced migration of colloids confined to a channel and subject to Poiseuille flow. We find that our approach reproduces qualitatively the main features of the Brownian dynamics density profiles, provided that the Péclet number input to the theory is treated as an adjustable parameter. It is likely that the agreement between the fitted value of Pe and the 'real' value employed in the Brownian dynamics simulations will improve when we consider smaller values of Pe. Nevertheless, it is quite gratifying that our theory can capture the qualitative form of the density profiles for this nontrivial outof-equilibrium problem.

In the near future we intend to improve further our theoretical approach, as well as to perform a more detailed comparison with Brownian dynamics simulation in order to better understand the range of validity of our various approximations. In particular it would be highly desirable to have exact solutions (or at least a more controlled approximation) for the distorted two-body density of colloids under Poiseuille flow. Further in this direction, it would also be of considerable interest to compare our present approach with that of Jin et al [36], with a view to improving our treatment of the distorted pair correlations in the presence of density inhomogeneities.

\section{Acknowledgments}

AS and JB thank the Swiss National Science Foundation for financial support. MK acknowledges support by Deutsche Forschungsgemeinschaft (DFG) grant No. KR 3844/2-1.

\section{Appendix A. Derivation of the radial equation}

Here we give a derivation of the differential equation (9) for determination of the radial function $h_{1}(r)$. We begin by collecting some useful identities. For a general second-rank tensor $\mathbf{M}$ the following holds

$$
\begin{gathered}
\nabla[(\hat{\mathbf{r}} \cdot \mathbf{M} \cdot \hat{\mathbf{r}}) a(r)]=\frac{2}{r^{2}}(\mathbf{r} \cdot \mathbf{M}) \cdot(\mathbf{1}-\hat{\mathbf{r}} \hat{\mathbf{r}}) a(r) \\
+\frac{1}{r}(\mathbf{r} \cdot \mathbf{M}) \cdot \hat{\mathbf{r}} \hat{\mathbf{r}} \frac{\mathrm{d} a(r)}{\mathrm{d} r}
\end{gathered}
$$

where $a(r)$ is an arbitrary radial function and $\hat{\mathbf{r}} \hat{\mathbf{r}}$ is a dyadic product. For an arbitrary vector field, $\mathbf{u}$, we have

$$
\nabla \cdot(\mathbf{u} \cdot \mathbf{M})=\mathbf{u} \cdot(\nabla \cdot \mathbf{M})+\mathbf{M}: \nabla \mathbf{u}
$$

where the double dot notation in the second term implies a full contraction, namely a scalar product followed by a trace operation. The divergence of a radial function mutiplied by $\mathbf{M}$ is

$$
\begin{aligned}
\nabla \cdot(a(r) \mathbf{M}) & =\nabla a(r) \cdot \mathbf{M}+\mathbf{a}(\mathbf{r}) \nabla \cdot \mathbf{M} \\
& =\left(\frac{\mathrm{d} a}{\mathrm{~d} r}\right) \hat{\mathbf{r}} \cdot \mathbf{M}+\mathbf{a}(\mathbf{r}) \nabla \cdot \mathbf{M} .
\end{aligned}
$$

For the special case $\mathbf{M}=r(\mathbf{1}-\hat{\mathbf{r}} \hat{\mathbf{r}})$, this reduces to

$$
\nabla \cdot(a(r) r(\mathbf{1}-\hat{\mathbf{r}} \hat{\mathbf{r}}))=-2 a(r) \hat{\mathbf{r}} .
$$

Finally we have the identity

$$
(\nabla(\mathbf{M} \cdot \hat{\mathbf{r}})) \cdot \hat{\mathbf{r}}=0
$$

and the elegant result

$$
\nabla \cdot(\mathbf{M} \cdot \hat{\mathbf{r}})=\frac{\operatorname{Tr}(\mathbf{M})}{r}-\frac{\hat{\mathbf{r}} \cdot \mathbf{M} \cdot \hat{\mathbf{r}}}{r} .
$$

Returning now to the physical problem of interest, we first note that for translationally invariant flow the velocity field can be written as $\mathbf{v}(\mathbf{r})=\mathbf{E} \cdot \mathbf{r}$. This suggests the following Ansatz for the solution of equation (9)

$$
g(\mathbf{r})=g_{0}(r)\left[1+A \frac{R^{2}}{D_{0}}(\hat{\mathbf{r}} \cdot \mathbf{E} \cdot \hat{\mathbf{r}}) h_{1}(r)\right]
$$

where $A$ is a dimensionless constant which remains to be determined. The expression is the most general form possessing the symmetry imposed by the external flow. Substitution of (A.8) into (9) and retaining only terms to leading order, yields for the steady state

$\nabla \cdot\left(\mathbf{v}(\mathbf{r}) g_{0}(r)\right)=2 R^{2} \nabla \cdot\left[A g_{0}(r) \nabla\left[(\hat{\mathbf{r}} \cdot \mathbf{E} \cdot \hat{\mathbf{r}}) h_{1}(r)\right]\right]$,

where we have used $\nabla g_{0}(r)=\beta(\nabla u(r)) g_{0}(r)$. Using identity (A.1) and the incompressibility condition, $\nabla \cdot \mathbf{v}=0$, enables us to re-write (A.9)

$$
\begin{aligned}
\frac{\hat{\mathbf{r}} \cdot \mathbf{E} \cdot \hat{\mathbf{r}}}{2 R^{2} A} r \frac{\mathrm{d} g_{0}(r)}{\mathrm{d} r}= & \nabla \cdot\left[\hat { \mathbf { r } } \cdot \mathbf { E } \cdot \left((\mathbf{1}-\hat{\mathbf{r}} \hat{\mathbf{r}}) \frac{2 g_{0}(r) h_{1}(r)}{r}\right.\right. \\
& \left.\left.+\hat{\mathbf{r}} \hat{\mathbf{r}} g_{0}(r) \frac{\mathrm{d} h_{1}(r)}{\mathrm{d} r}\right)\right] .
\end{aligned}
$$

Without loss of generality we fix the constant $A=-1 / 2$ and use the relation (A.3) to get

$$
\begin{aligned}
& -\frac{\hat{\mathbf{r}} \cdot \mathbf{E} \cdot \hat{\mathbf{r}}}{R^{2}} r \frac{\mathrm{d} g_{0}(r)}{\mathrm{d} r} \\
& =\hat{\mathbf{r}} \cdot \mathbf{E} \cdot\left(\nabla \cdot\left((\mathbf{1}-\hat{\mathbf{r}} \hat{\mathbf{r}}) \frac{2 g_{0}(r) h_{1}(r)}{r}+\hat{\mathbf{r}} \hat{\mathbf{r}} g_{0}(r) \frac{\mathrm{d} h_{1}(r)}{\mathrm{d} r}\right)\right) \\
& \quad+\left((\mathbf{1}-\hat{\mathbf{r}} \hat{\mathbf{r}}) \frac{2 g_{0} h_{1}(r)}{r}+\hat{\mathbf{r}} \hat{\mathbf{r}} g_{0}(r) \frac{\mathrm{d} h_{1}(r)}{\mathrm{d} r}\right): \nabla(\mathbf{E} \cdot \hat{\mathbf{r}}) .
\end{aligned}
$$

We will consider separately the two terms appearing in large brackets on the rhs of (A.11). To simplify term $(i)$ we use (A.4) and (A.5)) and obtain

$(i)=(\hat{\mathbf{r}} \cdot \mathbf{E} \cdot \hat{\mathbf{r}})\left(\frac{1}{r^{2}} \frac{\mathrm{d}}{\mathrm{d} r}\left(r^{2} g_{0}(r) \frac{\mathrm{d} h_{1}(r)}{\mathrm{d} r}\right)-4 g_{0} \frac{h_{1}(r)}{r^{2}}\right)$.

To simplify term (ii) we use (A.6) and (A.7) to obtain

$$
\begin{aligned}
(\text { ii })=\frac{2 g_{0}(r)}{r} \nabla \cdot(\mathbf{E} \cdot \hat{\mathbf{r}}) & =\frac{2 g_{0} h_{1}(r)}{r}\left(\frac{\operatorname{Tr}(\mathbf{E})}{r}-\frac{\hat{\mathbf{r}} \cdot \mathbf{E} \cdot \hat{\mathbf{r}}}{r}\right) \\
& =-\frac{2 g_{0} h_{1}(r)}{r^{2}}(\hat{\mathbf{r}} \cdot \mathbf{E} \cdot \hat{\mathbf{r}}),
\end{aligned}
$$


where $\operatorname{Tr}(\mathbf{E})=0$ has been used. Substituting (A.12) and (A.13) into (A.11) and introducing $\tilde{r}=r / R$ yields the desired result

$$
\begin{aligned}
(\hat{\mathbf{r}} & \cdot \mathbf{E} \cdot \hat{\mathbf{r}})\left(\frac{1}{\tilde{r}^{2}} \frac{\mathrm{d}}{\mathrm{d} \tilde{r}}\left(\tilde{r}^{2} g_{0}(r) \frac{\mathrm{d} h_{1}(r)}{\mathrm{d} \tilde{r}}\right)-6 g_{0}(r) \frac{h_{1}(r)}{\tilde{r}^{2}}\right) \\
& =-(\hat{\mathbf{r}} \cdot \mathbf{E} \cdot \hat{\mathbf{r}}) \tilde{r} \frac{\mathrm{d} g_{0}(r)}{\mathrm{d} \tilde{r}} .
\end{aligned}
$$

The coefficients of the quadratic form $(\hat{\mathbf{r}} \cdot \mathbf{E} \cdot \hat{\mathbf{r}})$ must be equal, because this expression remains valid for all choices of the traceless tensor E. We thus conclude that the Ansatz (A.8) is the correct leading order in Pe solution of the pair Smoluchowski equation (7), where $A=-1 / 2$ and the function $h_{1}(r)$ satisfies the differential equation (9). Similar calculations lead to the differential equations (12)-(15).

\section{Appendix B. Projecting the flow kernel in planar geometry}

We will concentrate first on the special case of shear flow, for which the flow kernel is given by (36). We select our planar geometry such that the density varies in the shear gradient direction, $\rho(\mathbf{r})=\rho(y)$, substitute (36) into (34) and perform the integrations in the $(x, y)$ plane. We employ a non-standard labelling for the spherical coordinate system, in order to be consistent with the usual choice (flow in $x$, gradient in $y$ ) employed in rheological studies. The angle $\theta$ is thus measured relative to the $y$-axis and the azimuthal angle $\phi$ relative to the $x$-axis. We thus seek to calculate the resolved kernel $\mathrm{K}(y)$, which is connected to the non-affine velocity field according to

$$
\mathbf{v}_{\mathrm{fl}}\left(y_{1}, t\right)=\int_{-\infty}^{\infty} \mathrm{d} y_{2} \rho\left(y_{2}\right) \mathrm{K}\left(y_{1}-y_{2}\right)
$$

\section{B.1. Hard spheres}

For the case of hard spheres the integration is restricted to the shell $r=2 R$. Integration in the $(x, y)$-plane thus corresponds to an integration over $\phi$ at fixed $y$ (fixed $\theta$ ). In the chosen coordinate system equation (36) becomes

$$
\begin{aligned}
\mathrm{K}^{\mathrm{hs}}(\theta, \phi)=-\hat{\mathbf{r}} & \frac{D_{0}}{R}\left(\operatorname{Pe} h_{1}(2 R) s(\theta) c(\theta) c(\phi)\right. \\
& -\operatorname{Pe}^{2}\left(h_{2}(2 R) s^{2}(\theta) c^{2}(\theta) c^{2}(\phi)\right. \\
& -h_{3}(2 R) \frac{\left(c^{2}(\theta)-s^{2}(\theta) c^{2}(\phi)\right)}{4} \\
& \left.\left.+h_{4}(2 R) \frac{\left(c^{2}(\theta)+s^{2}(\theta) c^{2}(\phi)\right)}{4}+\frac{1}{2} h_{5}(2 R)\right)\right) .
\end{aligned}
$$

where we use the shorthand notation $s(\cdot)=\sin (\cdot), c(\cdot)=\cos (\cdot)$. Substituting in the contact values of the $h$-functions for hard spheres

$$
\begin{aligned}
& h_{1}(2 R)=2 / 3 \\
& h_{2}(2 R)=0 \\
& h_{3}(2 R)=8 / 27 \\
& h_{4}(2 R)=-8 / 63 \\
& h_{5}(2 R)=-128 / 945
\end{aligned}
$$

then yields the kernel

$$
\begin{aligned}
\mathrm{K}^{\mathrm{hs}}(\theta, \phi)= & -\hat{\mathbf{r}} \frac{D_{0}}{R}\left[\frac{2}{3} \operatorname{Pe} c(\theta) s(\theta) c(\phi)\right. \\
& +\operatorname{Pe}^{2}\left(-\frac{2}{27}\left(s^{2}(\theta) c^{2}(\phi)-c^{2}(\theta)\right)\right. \\
& \left.\left.+\frac{2}{63}\left(s^{2}(\theta) c^{2}(\phi)+c^{2}(\theta)\right)+\frac{64}{945}\right)\right],
\end{aligned}
$$

The unit vector is given by

$$
\hat{\mathbf{r}}=s(\theta) c(\phi) \hat{\mathbf{e}}_{x}+c(\theta) \hat{\mathbf{e}}_{y}+s(\theta) s(\phi) \hat{\mathbf{e}}_{z} .
$$

Substitution of (B.4) into (B.3) and integration over $\phi$ yields for the $y$-component of the resolved kernel

$$
\begin{aligned}
\mathrm{K}_{y}^{\mathrm{hs}}(y)= & -\mathrm{Pe}^{2} \pi \frac{D_{0}}{R}\left(\frac{y}{2 R}\right)\left[-\frac{2}{27}\left(1-\frac{3 y^{2}}{(2 R)^{2}}\right)\right. \\
& \left.+\frac{2}{63}\left(1+\frac{y^{2}}{(2 R)^{2}}\right)+\frac{128}{945}\right]
\end{aligned}
$$

which is the component of physical interest for the present work.

\section{B.2. Hard sphere plus an attractive or repulsive tail}

We next consider the form of the projected kernel for potentials formed by the sum of a hard sphere repulsion and a longer range attractive (repulsive) tail, e.g. the hard-core Yukawa potential. For such potentials the kernel splits into a contact contribution and a tail contribution

$$
\mathrm{K}_{y}^{\mathrm{hst}}(y)=\mathrm{K}^{\mathrm{c}}(y) g_{\mathrm{eq}}(2 R)+\mathrm{K}_{y}^{\mathrm{t}}(y)
$$

where the first term is simply equation (B.5) scaled by the contact value of the equilibrium radial distribution function.

\section{Appendix C. Force balance and non-equilibrium osmotic pressure}

In this appendix, we repeat the arguments given in [7] to demonstrate that the approximations performed in the main text yield, for the case of a single wall, the exact value for the force exerted on the wall by the suspension. (Exact value in the sense that it equals the perpendicular pressure component in the bulk far way from the wall.) In orther words, our approximations correctly treat force balances. Let us therefore rewrite equation (1), using $\frac{\partial \rho(\mathbf{r}, t)}{\partial t}=-\nabla \cdot \mathbf{j}(\mathbf{r}, t)$, in terms of the stress in equation (24),

$$
\mathbf{j}(\mathbf{r}, \mathbf{t})=\rho(\mathbf{r}, t) \mathbf{v}(\mathbf{r})+\beta D\left(\nabla \cdot \boldsymbol{\sigma}-\nabla V_{\mathrm{ext}}\right) .
$$


Again, this equation is identical to equation (1) using equation (24) for the stress $\sigma$. For a steady state solution with a wall (hard or soft) being parallel to the $x z$ plane (compare figure 1), we have $\rho(\mathbf{r})=\rho(y)$, and

$$
j_{y}(y)=0 .
$$

As the quantities in equation (C.1) are invariant in $x$ and $z$ directions due to symmetry, equation (C.1) simplifies with equation (C.2) to

$$
-\int_{-\infty}^{\infty} \mathrm{d} y \rho(y) \partial_{y} V_{\mathrm{ext}}(y)=-\int_{-\infty}^{\infty} \mathrm{d} y \partial_{y} \sigma_{y y}(y) .
$$

The term on the lhs is identified as the force per surface area exerted on the external potential by the particles, $F_{y}^{\text {ext }} / A=-\int_{-\infty}^{\infty} \mathrm{d} y \rho(y) \partial_{y} V_{\text {ext }}(y)$. In case $V_{\text {ext }}$ grows sufficiently fast towards $y \rightarrow-\infty$ (e.g. in the case of an inpenetrable wall), we have vanishing density at $y=-\infty$, and hence

$$
\frac{F_{y}^{\mathrm{ext}}}{A}=-\sigma_{y y}(\infty)
$$

Equation (C.4) states the expected result that the yy component of the stress tensor in the bulk equals the force acting on the wall by the suspension, Actio et Reactio. However, is this true also for approximated solutions? As noted in [7], this is true for any approximation, that fulfills the following two conditions. (i) The approximation must conserve that the divergence of $\sigma$, with $\sigma$ given in equation (24) is indeed the expression entering the exact equation (1). This is mathematically true for any approxiamtion for $\rho^{(2)}$ in equation (1), which preserves the symmetry of $\rho^{(2)}$ in the two arguments $\mathbf{r}_{1}$ and $\mathbf{r}_{2}$. (ii) The approximation for $\rho^{(2)}$ must approach the correct result for the bulk. Both conditions are fulfilled by equation (30) for any pair potential and wall potential. We thus concluce that equations (30) and (31) give the correct force exerted on the wall (correct in the sense that it is as good as the approximations used for the bulk pair correlation).

\section{References}

[1] Dhont J K G 1996 An Introduction to Dynamics of Colloids (Amsterdam: Elsevier)

[2] Larson R G 1999 The Structure and Rheology of Complex Fluids (New York: Oxford University Press)

[3] Brader J 2010 J. Phys.: Condens. Matter 22363101
[4] Archer A J and Evans R 2004 J. Chem. Phys. 1214246

[5] Brader J M and Krüger M 2011 Mol. Phys. 1091029

[6] Krüger M and Brader J M 2011 Europhys. Lett. 9668006

[7] Aerov A A and Krüger M 2014 J. Chem. Phys. 140094701

[8] Chakrabarti J, Dzubiella J and Löwen H 2004 Phys. Rev. E 70012401

[9] Brady J F and Vicic M 1995 J. Rheol. 39545

[10] Blawzdziewicz J and Szamel G 1993 Phys. Rev. E 484632

[11] Evans R 1979 Adv. Phys. 28143

[12] Evans R 1992 Fundamentals of Inhomogeneous Fluids ed D Henderson (New York: Dekker)

[13] Rauscher M, Dominguez A, Krüger M and Penna F 2007 J. Chem. Phys. 127244906

[14] Krüger M and Rauscher M 2007 J. Chem. Phys. 127034905

[15] Krüger M 2006 Diploma Thesis University of Stuttgart, Germany

[16] Reinhardt J and Brader J M 2013 Europhys. Lett. 10228011

[17] Russell W B, Saville D A and Schowalter W A 1992 Colloidal Dispersions (Cambridge: Cambridge University Press)

[18] Swaroop M and Brady J F 2007 J. Rheol. 51409

[19] Kreutzer H J 1984 Non-Equilibrium Thermodynamics and its Statistical Foundations (Oxford: Oxford University Press)

[20] McQuarrie D A 2000 Statistical Mechanics (Mill Valley, CA: University Science Books)

[21] Hansen J-P and McDonald I R 1986 Theory of Simple Liquids (London: Academic)

[22] Mewis J and Wagner N J 2012 Colloidal Suspension Rheology (Cambridge: Cambridge University Press)

[23] Brady J F and Morris J F 1997 J. Fluid Mech. 348103

[24] Cichocki B 1987 Z. Phys. B 66537

[25] Bergenholtz J, Brady J F and Vicic M 2002 J. Fluid. Mech. 456239

[26] Semwogerere D and Weeks E R 2008 Phys. Fluids 20043306

[27] Kumar A and Graham M D 2012 Soft Matter 810536

[28] McCarthy K L and Kerr W L 1998 J. Food Eng. 3711

[29] Leighton D and Acrivos A 1987 J. Fluid. Mech. 181415

[30] Phillips R J et al 1992 Phys. Fluids A 430

[31] Nott P R and Brady J F 1994 J. Fluid Mech. 275157

[32] Morris J F and Brady J F 1998 Int. J. Multiph.Flow 24105

[33] Acrivos A, Mauro R and Fan X 1993 Int. J. Multiph. Flow 19797

[34] Frank M, Anderson D, Weeks E R and Morris J F 2003 J. Fluid. Mech. 493363

[35] Lyon M K and Leal L G 1998 J. Fluid. Mech. 36325

[36] Jin H, Ahn K H and Dhont J K G 2014 Soft Matter 109470

[37] Rosenfeld Y 1989 Phys. Rev. Lett. 63980

[38] Allen M P and Tildesley D J 1989 Computer Simulation of Liquids (Oxford: Clarendon) 